\title{
MicroRNAs: Potential Biomarkers and Targets of Therapy in Allergic Diseases?
}

\author{
Krzysztof Specjalski $^{1}$ [ $\cdot$ Ewa Jassem ${ }^{1}$
}

Received: 12 January 2019 / Accepted: 13 May 2019 / Published online: 28 May 2019

(c) The Author(s) 2019

\begin{abstract}
MicroRNAs (miRNAs) are small non-coding RNA molecules that are 18-22 nucleotides long and highly conserved throughout evolution. Currently, they are considered one of the fundamental regulatory mechanisms of genes expression. It has been demonstrated that miRNAs are involved in many biologic processes, such as signal transduction, cell proliferation and differentiation, apoptosis and stress responses. More recently, the role of miRNA has also been revealed in numerous immunological and inflammatory disorders, including allergic inflammation. Specific miRNA profiles were demonstrated in asthma, allergic rhinitis and atopic dermatitis. A core set of miRNAs involved in atopic diseases include upregulated miR-21, miR-223, miR-146a, miR-142-5p, miR-142-3p, miR-146b, miR-155 and downregulated let-7 family, miR-193b and miR-375. Most of the involved miRNAs increase secretion of Th2 cytokines (miR-1248, miR-146b), decrease secretion of Th1 cytokines (miR-513-5p, miR-625-5p) or promote differentiation of T cells towards Th2 (miR-21, miR-19a). In asthma miR-140-3p, miR-708 and miR-142-3p play a role in hyperplasia and hypertrophy of bronchial smooth muscle cells. Some single miRNAs or, more probably, their sets hold the promise for their use as biomarkers of atopic diseases. They are also promising target of future therapies.
\end{abstract}

Keywords miRNA $\cdot$ Allergy $\cdot$ Asthma $\cdot$ Allergic rhinitis $\cdot$ Atopic dermatitis

\section{Introduction}

MicroRNAs (miRNAs) are small non-coding RNA molecules that are 18-22 nucleotides long and highly conserved throughout evolution (Gebert and MacRae 2019). Their role remained unknown till 1990s when Victor Ambros discovered that lin-4 is a short RNA molecule blocking expression of the genes in the nematode Caenorhabditis elegans by RNA-RNA interaction (Lee et al. 1993). Currently, miRNA are considered one of the fundamental regulatory mechanisms of gene expression. Having bound to the region of 3' untranslated mRNA, miRNAs lead to decrease of gene expression by degradation of mRNA or inhibition of translation. Single miRNA can target numerous genes within one cell type and vice versa-the individual gene may be targeted by multiple miRNAs with only moderate effect of one miRNA on one target (Bartel 2009; Lu and Rothenberg

Krzysztof Specjalski

specjalski@gumed.edu.pl

1 Department of Allergology, Medical University of Gdańsk, Dębinki 7, 80-210 Gdańsk, Poland
2013). This makes relations between genes expression and miRNAs really complex. It has been demonstrated that miRNAs are involved in many biologic processes, such as signal transduction, cell proliferation and differentiation, apoptosis and stress responses. Their role in regulating of cell cycle was deeply investigated in oncology. For example, expression of several miRNAs was demonstrated to correlate with the histological type of lung cancer, prognosis and response to therapy (Skrzypski et al. 2014). More recently, the role of miRNA has also been revealed in numerous immunological and inflammatory disorders, including allergic inflammation. Recent studies have demonstrated specific miRNA profiles in some allergic conditions and asthma. Further, Rebane and Akdis (2014) have proposed that miRNAs contribute to the development of allergic diseases by influencing Th1/Th2 polarization, promoting chronic inflammation in epithelium and tissue remodelling as well as activating innate immune cells. In this review we discuss selected articles concerning miRNAs in allergic diseases in order to demonstrate role of miRNAs in the pathomechanism and possible application in monitoring and therapy. 


\section{Role of miRNAs in Allergic Diseases}

\section{Allergic Rhinitis}

Allergic rhinitis (AR) is the most common allergic disease worldwide with a prevalence of $1-40 \%$, depending on the region and population studied (Brożek et al. 2017). Although tendency to develop AR is inherited, environmental factors play a substantial role in the initiation of the disease. The key feature of AR is an IgE-mediated inflammation of nasal mucosa, characterized by eosinophilic infiltrations and Th1/Th2 cells imbalance. Th2 cytokines have been found essential in the pathomechanism of AR, particularly interleukin (IL)-4 inducing the subsets of Th2 cells and activating IgE production, IL-5 essential for eosinophils differentiation, maturation and survival, IL-13 playing a key role in mucus hypersecretion, inducing and maintaining $\operatorname{IgE}$ production and $\mathrm{IgE}-$ mediated responses. IL-1, IL-6 and tumour necrosis factor (TNF)- $\alpha$ secreted mainly by monocytes and macrophages also have a proinflammatory role, whereas transforming growing factor (TGF) $\beta$ and IL-10 have regulatory and anti-inflammatory effects (Wang et al. 2014; Eifan et al. 2015). As miRNAs exert well-known regulatory effects on functioning of immune system, their expression in AR has been investigated in recent years.

To analyse the impact of miRNA on cytokines production, Shaoqing et al. (2011) compared expression of 421 miRNAs in nasal mucosa of eight patients with perennial allergic rhinitis and eight healthy controls by means of real-time quantitative reverse transcriptase-PCR (qRTPCR) on microarrays (Exiqon). In AR group two miRNAs (miR-7 and miR-1194) were upregulated and seven downregulated (miR-224, miR-498, miR-187, miR-874, miR-143, miR-886, miR-767). Interestingly, in the previous studies downregulation of miR-224 was associated with inhibition of TGF- $\beta$ and miR-498 - with the functioning $\mathrm{CD}^{+} \mathrm{T}$ cells. Thus, the authors concluded that these miRNAs could play a role in development of AR. Suojalekto et al. (2013) investigated 159 young adults including patients with AR, AR and asthma, non-allergic rhinitis as well as healthy controls (life technologies microarrays). Out of 35 miRNAs analysed in nasal biopsies three, including above-mentioned miR-498 (miR-498, miR-155 and miR-205) were upregulated and one (let-7e) was downregulated in groups with AR. These changes correlated with increased levels of Th2 cytokines. Expression of miRNA in patients with non-allergic rhinitis patients did not differ from that in the healthy controls.

Members of let-7 family were shown to be decreased in both animal models and human studies on asthma and allergic rhinitis. On the contrary, their increased levels inhibit the expression of various inflammatory factors in AR by activating JAK1/STAT3 signalling pathway, repressing IL-13 production and inhibiting of SOCS4 gene transcription (Li et al. 2018).

Chen et al. (2010) investigated a panel of 157 miRNAs in mononuclear leukocytes from human umbilical cord blood with elevated IgE (applied biosystems microarrays). It was found that miR-21 and miR-126 were significantly lower in monocytes from children who developed allergic rhinitis. Downregulation of miR-21 and miR-126 was accompanied by upregulated expression of TGF- $\beta$ R 2 on monocytes. The findings were in line with earlier studies, where a significant increase of TGF- $\beta$ R2 in epithelium was revealed in patients with AR compared to healthy controls. Upregulation of miR21 was found to downregulate IL12A and TGF- $\beta$ R2 (Lu et al. 2009).

$\mathrm{Wu}$ et al. (2015) investigated expression of miRNAs in extracellular vesicles (exosomes) in nasal mucus in ten patients with severe AR and ten healthy controls by means of qRT-PCR (applied biosystems microarrays). From 366 tested miRNAs 21 were upregulated and 14 downregulated compared to healthy controls. Further analysis revealed that the processes influenced by differently expressed miRNAs involved B-cell receptor signalling pathway, the natural killer cell-mediated cytotoxicity, T-cell receptor signalling pathway, endocytosis, leucocyte transendothelial migration, etc. As transferring genetic material by exosomes to neighbouring cells modify their function, it seems that exosomal miRNAs form an intercellular signalling system. One of the most significantly upregulated molecules was miR-223 involved in eosinophilic inflammation and associated with lower regulatory T-cell numbers ( $\mathrm{Lu}$ et al. 2013a). It is well known that Treg cells play an important role in development of tolerance during immunotherapy by producing IL- 10 . Thus, in the future inhibition of miR-223 could be considered an adjuvant treatment during immunotherapy.

\section{Asthma}

Asthma is a chronic inflammatory disease, characterized by hyperresponsiveness and remodelling of the airways, affecting more than 300 million people worldwide (GINA Report 2018). The pathomechanism of this multifactorial disease is still not fully understood. The most common phenotype is eosinophilic inflammation associated with $\mathrm{Th} 2$ response and concomitant atopic diseases. However, some patients show neutrophilic, mixed type or paucigranulocytic pattern. The heterogeneity of asthmatic population is a serious limitation of several studies, because every conclusion should only refer to a single phenotype that was investigated and must not be generalized.

Increased serum levels of several miRNAs, including miR-16, miR-21, miR-125b, miR-126, miR-145, miR-146a, 
miR-148a, miR-221, miR-223, miR-338, miR-485-3p were demonstrated in general asthmatic populations (Liu et al. 2012; Qin et al. 2012; Panganiban et al. 2016; Lacedonia et al. 2017). Soujalehto et al. (2014) found downregulation of miR-18a, miR-126, let-7e, miR-155, miR-224 and upregulation of miR-498, miR-187, miR-874, miR-143, miR- 886$3 p$ in nasal biopsies in asthmatic patients with no differences between allergic and non-allergic phenotype. MiR-296-5p, miR-16-5p, miR-203 and miR-30d-5p were found to correlate with bronchial hyperresponsiveness while miR-155 with fractional exhaled nitric oxide, nasal nitric oxide and IgE (Davis et al. 2017). In mice model of asthma expression of miR-155, miR-21 and miR-18a correlated with Th2 cytokines levels in bronchoalveolar lavage fluid (Xiao et al. 2016). The significance of diverse expression of miRNAs in asthma is debatable. On one hand, Williams et al. (2009) found no difference in airway biopsies between healthy controls and patients with mild asthma, whereas Jardim et al. (2012) revealed 66 different expressions between asthmatics and controls in bronchial epithelial cells culture (including higher of miR-487b, miR-181, let-7f and lower of miR-203), with no relation to disease severity.

Single nucleotide polymorphisms (SNPs) in miRNAs genes may also play some role in the context of asthma development. Homozygous CC genotype of rs 2292832 $\mathrm{G} / \mathrm{C}$ (miR-149) has the highest frequency in asthmatics with coexisting allergic rhinitis. CC genotype of rs $2910164 \mathrm{G} / \mathrm{C}$ (miR-146a) and TT genotype in rs2292832 C/T (miR-149) are associated with decreased risk of asthma (Su et al. 2011; Hu et al. 2017).

The role of miRNAs in the pathomechanism of asthma could be analysed in three aspects-regulation of smooth muscle cells, epithelium and immune system.

\section{miRNAs in Smooth Muscle Cells}

In inflammatory diseases like asthma, smooth muscle cells (SMC) contribute to inflammation and airway wall remodelling by hypertrophy and hyperplasia. They are also capable of secreting cytokines and chemokines and have receptors for these mediators. Biomechanical properties of smooth muscle cells are apparently changed in asthmatic patients, suggesting that there is an asthmatic phenotype of these cells (Deshpande et al. 2015).

Recently, Alexandrova et al. (2016) performed a comprehensive analysis of small non-coding RNA expression in SMC in order to evaluate their permanent deregulation after asthma development. The expression of $905 \mathrm{miR}$ NAs in bronchial SMC was compared between asthmatics and non-asthmatics undergoing lung resection due to lung cancer. Expression of 40 miRNAs was significantly different between the groups, including nine upregulated and 31 downregulated. The authors found that 12 deregulated
miRNAs targeted 363 mRNAs expressed in SMC. The effect of targeting miRNAs was associated with significantly altered expression of 38 genes. The set included the gene encoding the peroxisome proliferator-activated receptor $\gamma$, which is known to be involved in airway inflammatory and remodelling responses (Oh et al. 2009). Another targets were: ITGB3 (integrin subunit $\beta 3$-playing a role in the pathogenesis of the disease and sensitization to allergens), BMPR2 (bone morphogenic protein receptor type 2-whose altered expression was shown to inhibit proliferation of vascular smooth muscle), PHB (prohibitin, which is responsible for reducing allergic airway inflammation) and adenosine A2b receptor (ADORA2B) - a powerful bronchoconstrictor acting as an inflammatory mediator and participating in airway wall remodelling (Berk et al. 2005; Weiss et al. 2005; Kariyawasam et al. 2008; Agrawal et al. 2012). Finally, PTEN (phosphatase and tensin homolog-regulating cell cycle, and glucocorticoid action during asthma treatment) was also influenced by miRNAs upregulation (Hosgood et al. 2009).

A few studies showed that miRNAs may influence three important component of SMC physiology (Table 1).

Further, in human airway smooth muscle cells exposed to cytokine stimulation mimicking airway conditions in asthma miR-145 was found to be highly expressed. Blocking miR-145 with anti-miR resulted in decreased proliferation and migration of SMC in dose-dependent manner, as well as decreased synthesis of collagen I and myosin

Table 1 Influence of selected miRNAs on bronchial smooth muscle cells (SMC) in asthma

\begin{tabular}{|c|c|c|}
\hline Altered miRNA & Target & SMC function \\
\hline $\begin{array}{l}\text { miR-140-3p } \\
\text { miR-708 (Deshpande et al. } \\
\text { 2005a, b) } \\
\text { miR-10 (Hu et al. 2014) } \\
\text { miR-142-3p (Bartel et al. 2018) }\end{array}$ & $\begin{array}{l}\text { CD38 }{ }^{\mathrm{a}} \\
\text { p13 kinase } \\
\text { WNT pathway }\end{array}$ & $\begin{array}{l}\text { Hyperplasia } \\
\text { Hypertrophy } \\
\text { Remodelling }\end{array}$ \\
\hline miR-25 (Kuhn et al. 2010) & $\begin{array}{l}\text { Eotaxin } \\
\text { RANTES }\end{array}$ & $\begin{array}{l}\text { Secretion of } \\
\text { cytokines } \\
\text { and } \\
\text { chemokines }\end{array}$ \\
\hline miR-133 (Chiba et al. 2009) & $\begin{array}{l}\text { IL-13 } \\
\text { GTPase RhoA }\end{array}$ & Contraction \\
\hline
\end{tabular}

WNT Wingless/Integrase 1

${ }^{\mathrm{a}} \mathrm{CD} 38$ is a cell surface regulating calcium concentration and contractility of SMC. It also plays a role in innate immunity and is involved in T- and B-cell function and immune responses to antigen. In mice model CD $38^{-/-}$organisms did not develop "asthmatic phenotype" following IL-13 and TNF- $\alpha$ challenge

${ }^{b}$ Exposure of smooth muscles on IL-13 and downregulation of miR133a leads to upregulation of GTPase RhoA. RhoA is involved in contraction of SMC and is significantly augmented in asthma. Thus, miR-133a is regarded as one of the regulators of airway hyperresponsiveness 
heavy chain. The target of miR-145 is Kruppel-like factor 4 (KLF4), a member of KLF transcription factor family. Inhibition of miR-145 increases levels of KLF4 and decreases matrix metalloproteinases (MMP)-2 and MMP-9 (Liu et al. 2015).

Let-7 family has been found to be downregulated in allergic diseases. In asthma this group of molecules plays a substantial role by regulating $\beta 2$ receptors. Lower expression of let-7f attenuates receptor downregulation (Wang et al. 2011).

\section{Functional Role of miRNAs in Epithelium}

The bronchial epithelium is considered a physical protection against pathogens. In fact it is also an immunity organ secreting cytokines and regulating inflammatory process in asthma. Asthmatic patients show upregulation of miR-19a and downregulation of miR-181-5p in bronchial epithelium. The former is related to cells proliferation through targeting TGF- $\beta$ R 2 mRNA (Haj-Salem et al. 2015). The latter inversely correlates with sputum and bronchial submucosal eosinophilia. The target of miR-181-5p is SPP1, gene encoding osteopontin, what leads to regulation of IL13, IL-1 $\beta$ and CCL11 expression (Huo et al. 2016).

Solberg et al. (2012) compared expression of miRNAs in bronchial epithelium in 16 steroid-naïve asthmatics, 19 steroid-using asthmatics and 12 healthy controls. Twelve out of 16 steroid-naïve patients had different expression pattern of 217 miRNA compared to healthy controls including members of miR-34/449 family (miR-34b-5p and $\mathrm{miR}-34 \mathrm{c}-5 \mathrm{p}$ ) repressed by IL-13. Moreover, in steroid-naïve subjects with asthma, standard treatment with inhaled glucocorticosteroids had only modest effect on miRNA expression.

\section{The Role of miRNAs in Eosinophilic Asthma}

Eosinophilic asthma results from Th2 type inflammation ("T2-high asthma"). As a consequence, miRNAs associated with secretion of Th2-related cytokines (IL-4, IL-5, IL-13) or activity of T cells and B cells are dysregulated (Table 2).

Eosinophils are key cells in T2-high asthma. In ex vivo culture model of murine bone marrow-derived eosinophils miR-21 and miR-223 are upregulated (Lu et al. 2013a, b). MiR-21-deficient eosinophil progenitors have an increased apoptosis rate during maturation. MiR-21-deficient mice have reduced eosinophil levels in blood. In contrast, miR223-deficient eosinophil progenitors proliferate more intensively.

\section{The Role of miRNAs in Neutrophilic Asthma}

One of the most significant challenges in asthma is neutrophilic endotype. So far, no biomarkers are available to confirm the diagnosis and monitor the disease. The routine asthma treatment is less efficient and the course of the disease is more severe.

In mice model of neutrophilic, infection-induced asthma Kim et al. (2017) found upregulation of miR-21 in homogenized lungs. The authors demonstrated that miR-21 promotes PI3K-mediated phosphorylation and nuclear translocation of pAKT that suppresses HDAC2 levels and leads to steroid insensitivity.

In human neutrophilic asthma upregulation of miR199a-5p, miR-223-3p, miR-142-3p and miR-629-3p was

Table 2 Influence of selected miRNAs on immune system in asthma

\begin{tabular}{|c|c|c|}
\hline Altered miRNA & Target & Function \\
\hline miR-155 (Zhou et al. 2016a; Zhang et al. 2017) & $\begin{array}{l}\text { IL-4 } \\
\text { IL-5 } \\
\text { IL-13 } \\
\text { IL-17a } \\
\text { CTLA-4 } \\
\text { CD4 }{ }^{+}\end{array}$ & $\begin{array}{l}\text { Enhanced inflammation and mucus secretion } \\
\text { Regulation of T-cell activation } \\
\text { Influence on proliferative response }\end{array}$ \\
\hline miR-210 (Long et al. 2017) & & Inhibition of Treg function \\
\hline $\operatorname{miR}-181 \mathrm{a}$ & & Augmenting sensitivity of $\mathrm{T}$ cells to peptide antigens \\
\hline $\begin{array}{l}\text { miR-21, miR-19a (Li et al. 2007; Lu et al. 2009, 2011; } \\
\text { Sawant et al. 2013; Long et al. 2017) }\end{array}$ & & Promoting differentiation of $\mathrm{T}$ cells towards $\mathrm{Th} 2$ \\
\hline miR-221-3p (Zhou et al. 2016b) & PTEN & IL-4 upregulation \\
\hline $\begin{array}{l}\text { miR-1248 } \\
\text { miR-146a (Panganiban et al. 2012; Yang et al. 2017) }\end{array}$ & & $\begin{array}{l}\text { IL-5 upregulation } \\
\text { IL-5 inhibition }\end{array}$ \\
\hline let-7 family (Polikepahad et al. 2010; Kumar et al. 2011) & & IL-13 downregulation \\
\hline $\begin{array}{l}\text { miR-323-3p, miR-181a, miR-26a (Karner et al. 2017) } \\
\text { miR-513-5p, miR-22-3p, miR-625-5p (Dong et al. 2016) }\end{array}$ & SMAD2, SMAD3 & $\begin{array}{l}\text { TGF- } \beta \text {-dependent signalling pathway modulation } \\
\text { Inhibition of Th } 1 \text { cytokines including IL- } 12 \text {, and interferon- } \gamma\end{array}$ \\
\hline
\end{tabular}


found in induced sputum. MiR-629-3p is expressed in bronchial epithelium and miR-223-3p and miR-142-3p-in neutrophils, monocytes and macrophages. Level of miR$199 a-5 p$ was significantly associated with impaired pulmonary function (Huang et al. 2018). MiR-629-3p induces upregulation of IL-8 in epithelium, thus providing a mechanistic link between increased miR-629-3p expression and airway neutrophilia in patients with severe asthma (Maes et al. 2016).

\section{Atopic Dermatitis}

Atopic dermatitis (AD) is an inflammatory disease of the skin characterized by pruritus as well as typical appearance and distribution of the rash, which follows a chronic relapsing and remitting course. Atopic dermatitis is one of the most common skin diseases affecting up to $20 \%$ of children and $2-8 \%$ of the adults (Odhiambo et al. 2009; Wollenberg et al. 2018). Approximately $40-60 \%$ of patients have concomitant atopic comorbidities including asthma, allergic rhinitis or food allergies (Flohr et al. 2004). Development of $\mathrm{AD}$ results from the interaction of numerous genetical and environmental factors. Genetic studies identified a central role of genes associated with skin barrier impairment. Particularly, filaggrin gene mutations resulting in loss of its function are correlated with early onset, more severe lesions and higher number of comorbidities (Brown 2017). On the other hand, immune dysfunction with Th2-mediated inflammation is a crucial phenomenon which recently has become a target for successful biological therapies. In acute lesions, $\mathrm{AD}$ is characterized by profound increases of Th2 cells and Th2-related cytokines (IL-4, IL-5, IL-13, IL-31) as well as IL-22 and Th22 cells (Brunner et al. 2017).

Despite clinical significance of $\mathrm{AD}$, there is less literature data on the role of miRNAs in the mechanism of the disease compared to that for asthma or allergic rhinitis. Most of the studies confirm upregulation of miRNAs known for their involvement in asthma or allergic rhinitis (miR-21, miR146a, miR-203) (Wu et al. 2015). Other miRNAs, like miR720 , are upregulated solely in $\mathrm{AD}$, possibly due to their role in organ-specific functions, such as keratinocyte cell cycle regulation (Chikh et al. 2011). MiR-483-5p was found to be upregulated in the sera of children with $\mathrm{AD}$, but only in cases with other concomitant atopic diseases ( $\mathrm{Lv}$ et al. 2014). In skin with AD lesions miR-155 was highly upregulated (Sonkoly et al. 2010). Cytotoxic T lymphocyte-associated protein (CTLA)-4, major inhibitory molecule of T-cell responses, was identified as a target for miR-155. Blockade of CTLA-4 maintains or increases allergic responses with eosinophilia and elevated $\operatorname{IgE}$ levels, while increased CTLA-4 inhibits allergic inflammation (Hellings et al. 2002; Jen et al. 2007). Interestingly, early development of AD in children was found to be associated with the high expression of miR-146b, miR-21, miR-22 and miR-375 in their mothers' breast milk (Simpson et al. 2015). This is in line with clinical data indicating high prevalence of atopic disease in the families of children with AD. Given the fact that breast milks miRNAs are stable and biologically active after ingestion the authors have also hypothesized that miRNAs could affect infants' immune system and regulate maturation of gastrointestinal tract. In genetically predisposed individuals exposure to "pro-allergic" miRNAs could facilitate development of AD.

\section{MiRNAs as Biomarkers}

Allergic diseases are complex, with numerous genetic and inflammatory pathways involved and many phenotypes. In vast majority of cases IgE-mediated allergy can be confirmed with standard tools (skin tests, sIgE measurement, provocation tests) or some novel methods developed in recent years such as basophil activation test or componentresolved diagnostics (Heinzerling et al. 2013; Borres et al. 2016; Hemmings et al. 2018). However, in many cases diagnosis is based on subjective, observational criteria, for example Hanifin and Rajka criteria for atopic dermatitis requiring long-term observation for confirmation of diagnosis. In asthma the diagnosis in based on clinical picture and presence of expiratory airflow limitation variable over time. However, in small children pulmonary function tests are often difficult to perform and diagnosis remains unconfirmed. Moreover, a quick and simple endotyping of individual asthmatic patients is still unmet need that could make it possible to tailor the treatment properly and increase its efficacy. So far, some of the phenotypes of asthma, e.g., neutrophilic, do not have well established biomarkers. Thus, there are objectives not sufficiently achievable with the tools currently applied in clinical practice. Biomarkers facilitating diagnosing of these diseases, their endotyping and/or predicting clinical outcomes and drug response would be a big step forward to reach these goals. The most significant disadvantage of applying miRNAs is their specificity which is uncertain due to potential role in pathomechanism of several diseases.

The ideal biomarker should have good performance characteristics, such as sensitivity, specificity and positive and negative predictive values. Moreover, it should be relatively safe, easy to measure and cost-effective (Parikh and Vasan 2007). Thus, apart from scientific evidence confirming that its use influences disease diagnosing or outcome, the biomarker should be easily applied in the clinical practice. In some aspects miRNAs seem to be a promising direction in this field.

As mentioned above, allergic rhinitis is characterized by typical profiles of miRNAs expression. Among numerous 
candidates for biomarkers of AR, miR-181a seems to be particularly promising. The serum level of this miRNA is significantly decreased in patients with $\mathrm{AR}$, and its expression negatively correlates with disease severity, levels of osteopontin, Th2 cytokines (IL-4, IL-5) and positively correlates with Th1 cytokines (IL-12, interferon $\gamma$ ) (Liu et al. 2016b). However, due to complex relations between miRNAs and inflammatory pathways in AR, expression of single miRNA may not be a truly reliable biomarker. Thus, several attempts were made to find a model based on a group of selected miRNAs that correlate with the presence and severity of AR. For example, a combination of miR-126-5p, miR-19a-5p and miR-26a-5p could confirm diagnosis of AR with sensitivity of $90 \%$ and specificity of $70 \%$ and correlates with disease severity (Jia et al. 2018). In other study, seven candidate miRNAs were selected based on earlier studies and investigated in 85 patients with AR and 57 non-atopic patients after adenoid surgery (He et al. 2017). Expression of miR-221 and miR-142-3p was found to be elevated and combination of their levels could confirm diagnosis of AR with the sensitivity of $81 \%$ and specificity of $65 \%$. Expression of miR221 also correlated positively with clinical data (total nasal score, itching and sneezing scores). To conclude, currently diagnosis of allergic rhinitis is based on patients' history, skin tests and/or IgE determination. miRNAs determination will surely not replace this but the future studies can prove their value in assessment of severity and prognosing.

Asthma is a heterogeneous disease with phenotypes that may react differently to therapy, so there is an apparent need for novel tools to differentiate patients into several subgroups. Milger et al. (2017) pre-identified miRNA biomarkers in murine models of asthma, and candidate biomarkers were confirmed in a test cohort of patients with asthma and healthy controls. Although the observed differences in the single miRNAs were considered small, the combination of five miRNA ratios (miR-21-5p/miR-15a-5p; miR27a-3p/miR-15a-5p; miR-29c-3p/miR15a-5p; miR-223-3p/ miR-425-5p; miR-15a-5p/miR342-3p) was found a reliable asthma biomarker. However, with the use of these biomarkers the authors were not able to determine particular asthmatic phenotypes. Although patients taking oral steroids or receiving antileukotriene therapy had different expression, inflammatory phenotype was not reflected. Panganiban et al. (2016) compared patients with asthma, AR and healthy controls. They found 30 miRNAs with expression significantly different between the groups. Specific for asthma was upregulation of miR-16, miR-223, miR-148a and miR-146a, and downregulation od miR-299-5p, miR-570 and miR-150. The model with six most relevant miRNAs (miR-125b, miR-16, miR-299-5p, miR-126, mir-206, miR-133b) allowed differentiation of healthy subjects, AR or asthma with the sensitivity of $92.4 \%$. It was also possible to find two expression profiles corresponding with peripheral eosinophil levels.
One of the most important challenges would be to find biomarkers predicting treatment outcomes. McGeachie et al. (2017) investigated serum expression of 738 miRNAs in 160 children with asthma aged 5-12 years in search for predictors of asthma remission at the age of 14 . The developed model based on 12 variables (including miR-146b-5p, miR-106a, miR-126, miR-30a) allowed prediction of remission with sensitivity of $84 \%$ and specificity of $70 \%$. Thus, miRNAs are potentially predictive biomarkers for treatment outcomes.

Finally, Trinh et al. (2017) investigated SNPs in miR196a (rs11614913 T/C), miR-146a (rs2910164 C/G) and miR-499 (rs3746444 A/G) in 347 Korean asthma patients and 172 healthy controls. The CT/CC genotype of miR196a rs11614913 was associated with eosinophilic asthma $(p=0.004)$ and a higher sputum eosinophil count compared with the TT genotype $(p=0.003)$. The CG/GG genotype of miR-146a rs2910164 tended to be associated with higher bronchial hyperresponsiveness to methacholine $\left(\mathrm{PC}_{20}\right)$ compared with the $\mathrm{CC}$ genotype. The AG/GG genotype of miR499 rs3746444 was associated with higher predicted values of forced expiratory volume in $1 \mathrm{~s}\left(\% \mathrm{FEV}_{1}\right)$ compared with the AA genotype $(p=0.008)$.

The presented studies concentrated mostly on finding miRNAs typical for asthma. As this is a heterogenous disease, it would be beneficial to find miRNAs associated with its phenotypes. So far, this is an unmet need. Differentiating between asthma phenotypes on the basis of miRNAs would have practical application in the future.

Another clinically significant application of miRNAs may be associated with their potential predictive value in allergen immunotherapy. Allergen immunotherapy is a method of tolerance induction by administration of increasing doses of allergen to which the patient has IgE-mediated allergy. Currently in clinical practice response to allergen immunotherapy is based mainly on observation of clinical symptoms. For scientific purposes challenge tests are also applied. Although reliable, they are time-consuming and potentially dangerous for the patients, because they involve exposure to clinically relevant allergens. Determination of miRNAs profiles during immunotherapy would provide an insight into its genetic consequences. Thus, some studies have been conducted to assess whether miRNAs monitoring could be an objective method of evaluating the response and prognosing.

In the study of Specjalski et al. (2016) expression of 740 miRNAs was assessed before and $24 \mathrm{~h}$ after initial phase of allergen immunotherapy with wasp venom. Expression of five miRNAs changed significantly (miR-370, miR539, miR-502-3p, miR-299 and miR-29c), and another 62 changed twofold in some patients, including increases in miR-143, let-7d and decreases in proinflammatory miR-301, miR-146b, miR-106, miR-485. In the study of Luo et al. (2016) 24 children with AR and house dust mites allergy 
were treated either with sublingual (SLIT) or subcutaneous immunotherapy (SCIT). In both groups after 3 months of treatment increased expression of miR-146a correlated positively with Foxp3 mRNA level and negatively with disease severity. Increased IL-10 and decreased of IL-5 levels were also found with no differences between SLIT and SCIT. In none of these studies changing expression of miRNAs was confronted with clinical response to immunotherapy. Such assessment was made by Hou et al. (2015). The authors investigated miRNAs profile in patients with allergy to Japanese cedar pollen undergoing SLIT. Out of 15 patients, six underwent sublingual immunotherapy with the pollens and nine received placebo. During pollinating season seven subjects in the placebo group and none administered SLIT developed symptoms of rhinitis, respectively. Sera miRNAs levels were determined in pre- and post-seasonal samples. The only miRNAs that changed significantly in both, SLIT and placebo group, was miR-223. In placebo group development of pollinosis was significantly associated with downregulation of let- $7 \mathrm{~b}$. Although monitoring of immunotherapy with reliable biomarker would be useful, currently it seems that no miRNAs could be applied in this setting.

\section{MiRNAs as Therapeutic Target}

MiRNAs have been assessed as therapeutic agents in several in vitro and animal studies. Although their results seem promising, effects of therapy with miRNAs or their antagomirs are difficult to predict. Relations between gene expression and miRNAs are complex. As a result, administration or silencing of a single miRNA could modify expression of numerous genes with unknown consequences. In multifactorial diseases, successful silencing of a single gene may not be efficient in clinical practice. On the other hand, miRNA are still promising therapeutic agents due to the site-specificity which is usually associated with the reduction of side effects. However, the potential of miRNAs-based therapeutics has not been fully applied due to inefficient transportation of miRNA to the target cells. This requires carriers (e.g., viral or lipid-based vectors) which are now extensively investigated.

In allergic diseases, the crucial processes influenced by miRNAs include eosinophils development, T cells differentiation and activation and development and activation of mast cells. Thus, they may be considered as a potential means of modulating gene expression to reduce Th2 inflammation.

In in vitro studies miR-106b was found to negatively regulate pro-allergic properties of dendritic cells, whereas and miR-143, which is downregulated in AR, suppressed IL-13 receptor $\alpha 1$ chain gene expression in nasal epithelial cells (Yu et al. 2013; Tang et al. 2015; Teng et al. 2015). MiR30a-5p targeted suppressor of cytokine signalling 3, playing a role in $\mathrm{T}$ helper cell differentiation and involved in the development of AR (Zhao et al. 2018). Administration of miR-375 agomir, by inhibiting JAK2/STAT3 pathway, led to downregulation of IL-6 and TNF- $\alpha$ and upregulation of IL-10 in nasal mucosa cells (Wang et al. 2018).

In animal models, effects of miRNAs administration were investigated mostly in the context of AR. Deng et al. (2015) investigated the influence of miR-135a, which had been known to downregulate GATA-3, playing an important role in mast cells degranulation. MiR-135a was administered intranasally to ovalbumin (OVA)-sensitized mice with the use of lentiviral vector. Following the treatment, infiltrations with eosinophils and mast cells were suppressed in nasal mucosa and Th1/Th2 cells balance in the spleen shifted towards Th1 dominance. Similar effects were observed in the study of Luo et al. (2014). Intranasal application of miR135a in OVA-sensitized mice lowered expression of both GATA-3 and IL-4. Xiao et al. (2017) found that expression of miR-133b was significantly decreased in nasal mucosa of mice with AR. Its restoration by nasal administration of miR-133b agomir led to amelioration of rhinitis symptoms and decreasing of serum levels of IgE, IL-4, IL-5 and TNF$\alpha$. Additionally, miR-133b appeared to attenuate eosinophils and mast cells infiltration in nasal mucosa.

In murine model of asthma Niu et al. (2017) tested effects of miR-33b known for its regulatory role in mediating mast cell function. The study demonstrated that several key elements of asthma, including infiltration of inflammatory cells, especially eosinophils, as well as Th2 cytokines in bronchoalveolar lavage fluid, airway hyperresponsiveness and specific IgE levels were reduced with miR-33b. Thus, miR-33b-mediated inhibition of asthma development was associated with Th2 response inhibition.

Liu et al. (2016a) investigated the role of miR-146a in an enforcing immunotherapy in a mice model of AR. MiR146a was shown to be downregulated in both humans and mice sensitized to OVA. Sensitized mice with AR symptoms were administered intranasal immunotherapy with nanovaccine (i.e., vaccine containing nanoparticles as carriers or adjuvants) containing either OVA, miR-146a or both (Hayat and Darroudi 2019). The study showed that nanovaccine with ovalbumin exacerbated AR symptoms, whereas there was no impact of miR-146a. However, adding miR-146a to ovalbumin significantly enforced the effect of immunotherapy, leading to inhibition of AR symptoms and increase of Treg cells in nasal mucosa. The effect was at least partly associated with the induction of TGF- $\beta$, which is of crucial importance in immunotherapy and induction of IL-10 suppressing allergic reaction in nasal mucosa (Liu et al. 2016a; Luo et al. 2015).

Another possible therapeutic option are antagomirs. They are a class of chemically engineered antisense oligonucleotides complementary to the specific miRNA target. 
They bind to both miRNAs and miRNAs binding site of mRNA targets. As a consequence, they sequester miRNA in competition with cellular target mRNA leading to functional inhibition of the miRNA and derepression of the direct targets. Depending on antagomir chemistry miRNA is either sequestered in heteroduplex or its degradation is promoted (Stenvang et al. 2012). So far their application has been investigated in animal models. In mice, anti-miR-221 reduced eosinophils in bronchoalveolar lavage (Qin et al. 2012). Anti-miR-145 and anti-miR-126 reduced eosinophilic infiltrations in bronchi, Th2 cytokines production and airway hyperreactivity (Collison et al. 2011; McClure et al. 2014). By contrast, such results were not achieved with anti-miR$155-5 \mathrm{p}$. Although miR-155-5p was highly upregulated in murine asthma, intranasal administration of antagomir had no effect on airway hyperresponsiveness or Th2 cytokines expression (Plank et al. 2015).

In humans some phase I clinical trials with miRNAs and antagomirs have been conducted in recent years. Miravirsen (anti-miR-122) tested in hepatitis C was found to be efficient in decreasing HCV load (Janssen et al. 2013). MiR-16 was tested in mesothelioma and miR-34 in multiple solid tumours. Both were found safe and will be assessed in next phase trials (Hong et al. 2015; Reid et al. 2016).

In relation to allergic diseases the unanswered questions concerning therapy with miRNAs include defining groups of potential good responders, optimal route of administration and safety assessments.

\section{Conclusions}

miRNAs have an unquestionable influence on regulation of allergic inflammation. A core set of miRNAs involved in atopic diseases include upregulated miR-21, miR-223, miR-146a, miR-142-5p, miR-142-3p, miR-146b, miR-155 and downregulated let-7 family, miR-193b and miR-375. Some single miRNAs or, more probably, their sets hold the promise for their use as biomarkers of atopic diseases. They are also promising target of future therapies.

Although our knowledge about role of miRNAs in allergic inflammation has expanded in recent years, several areas warrant future investigation. Notably, except for a few early studies in human asthma or rhinitis, most of the knowledge on the role of miRNAs in allergic inflammation is based on cell cultures and murine models. Thus, future studies should be more focused on human settings. Application of miRNAs as non-invasive biomarkers should be investigated with an emphasis of possible determination of disease endotype and predicting treatment effects. Another aspect is the possible therapeutic application. Considering complex relations between miRNAs and genes with Th1/Th2 balance regulated by numerous miRNAs, results of animal studies cannot be easily translated to the clinics. Combinatorial targeting of a key RNA by several miRNAs would be a promising area of future investigation.

Acknowledgements This study was supported by Medical University of Gdańsk (ST-554).

\section{Compliance with Ethical Standards}

Conflict of interest The authors declare that they have no conflict of interest.

Open Access This article is distributed under the terms of the Creative Commons Attribution 4.0 International License (http://creativeco mmons.org/licenses/by/4.0/), which permits unrestricted use, distribution, and reproduction in any medium, provided you give appropriate credit to the original author(s) and the source, provide a link to the Creative Commons license, and indicate if changes were made.

\section{References}

Agrawal T, Gupta G, Agrawal D (2012) Vitamin D deficiency decreases the expression of VDR and prohibitin in the lungs of mice with allergic airway inflammation. Exp Mol Pathol 93:74-81

Alexandrova E, Miglino N, Hashim A et al (2016) Small RNA profiling reveals deregulated phosphatase and tensin homolog (PTEN)/ phosphoinositide 3-kinase (PI3 K)/Akt pathway in bronchial smooth muscle cells from asthmatic patients. J Allergy Clin Immunol 137:58-67

Bartel D (2009) MicroRNAs: target recognition and regulatory functions. Cell 136:215-233

Bartel S, Carraro G, Alessandrini F et al (2018) MiR-142-3p is associated with aberrant Wingless/Integrase I (WNT) signaling during airway remodeling in asthma. Am J Physiol Lung Cell Mol Physiol 315:L328-L333

Berk B, Akgun H, Erol K et al (2005) New 8-substituted xanthiene derivates as potent bronchodilators. Farmaco 60:974-980

Borres M, Maruyama N, Sato S et al (2016) Recent advances in component resolved diagnosis in food allergy. Allergol Int 65:378-387

Brown S (2017) Molecular mechanisms in atopic eczema: insights gained from genetic studies. J Pathol 241:140-145

Brożek JL, Bousquet J, Agache I et al (2017) Allergic rhinitis and its impact on asthma (ARIA) guidelines-2016 revision. J Allergy Clin Immunol 140:950-958

Brunner PM, Guttman-Yassky E, Leung DY (2017) The immunology of atopic dermatitis and its reversibility with broad-spectrum and targeted therapies. J Allergy Clin Immunol 139(4S):S65-S76

Chen R, Huang H, Ou C et al (2010) MicroRNA-21 expression in neonatal blood associated with antenatal immunoglobulin E production and development of allergic rhinitis. Clin Exp Allergy 40:1482-1490

Chiba Y, Tanabe M, Goto K et al (2009) Downregulation of miR-133a contributes to up-regulation of Rhoa in bronchial smooth muscle cells. Am J Respir Crit Care Med 180:713-719

Chikh A, Matin R, Senatore V et al (2011) iASPP/p63 autoregulatory feedback loop is required for homeostasis of stratified epithelia. EMBO J 30:4261-4273

Collison A, Herbert C, Siegle J et al (2011) Altered expression of microRNA in the airway wall in chronic asthma: miR-126 as a potential therapeutic target. BMC Pulm Med 11:29 
Davis J, Sun M, Kho A et al (2017) Circulating microRNAs and association with methacholine PC20 in the childhood asthma management program cohort. PLoS One 12:e0180329

Deng Y, Yang Y, Wang S et al (2015) Intranasal administration of lentiviral miR-135a regulates mast cell and antigen-induced inflammation by targeting GATA3. PLoS One 10:e0139322

Deshpande DA, White TA, Dogan S et al (2005a) CD38/cyclic ADPribose signaling: role in the regulation of calcium homeostasis in airway smooth muscle. Am J Physiol Lung Cell Mol Physiol 288:L773-L788

Deshpande DA, White TA, Guedes AG et al (2005b) Altered airway responsiveness in CD38-deficient mice. Am J Respir Cell Mol Biol 32:149-156

Deshpande DA, Dileepan M, Walseth TF et al (2015) MicroRNA regulation of airway inflammation and airway smooth muscle function: relevance to asthma. Drug Dev Res 76:286-295

Dong X, Xu M, Ren Z et al (2016) Regulation of CBL and ESR1 expression by microRNA-22-3p, 513a-5p and 625-5p may impact the pathogenesis of dust mite-induced paediatric asthma. Int J Mol Med 38:446-456

Eifan A, Orban N, Jacobson M et al (2015) Decreased expression of nasal tissue TGF-beta may contribute to allergic inflammation in allergic rhinitis but not remodelling. Allergy Suppl 101:628

Flohr C, Johanson S, Wahlgren C et al (2004) How atopic is atopic dermatitis? J Allergy Clin Immunol 114:150-158

Gebert L, MacRae IJ (2019) Regulation of microRNA function in animals. Nat Rev Mol Cell Biol 20:21-37

Global Initiative for Asthma (2018) Report - Global Strategy for Asthma Management and Prevention. https://ginasthma.org. Accessed 3 Jun 2018

Haj-Salem I, Fakhfakh R, Berube J et al (2015) MicroRNA-19a enhances proliferation of bronchial epithelial cells by targeting $T G F \beta R 2$ gene in severe asthma. Allergy 70:212-219

Hayat S, Darroudi M (2019) Nanovaccine: a novel approach in immunization. J Cell Physiol 234:12530-12536

He P, Ni J, Zhao H et al (2017) Diagnostic value of miR-221 and miR-142-3p expressions of allergic rhinitis and miR-221 level is positively correlated with disease severity. Int J Clin Exp Med 10:7834-7842

Heinzerling L, Mari A, Bergmann KC et al (2013) The skin prick testEuropean standards. Clin Transl Allergy 3:3

Hellings PW, Vandenberghe P, Kasran A et al (2002) Blockage of CTLA-4 enhances allergic sensitization and eosinophilic airway inflammation in genetically predisposed mice. Eur J Immunol 32:585-594

Hemmings O, Kwok M, McKendry R et al (2018) Basophil activation test: old and new applications in allergy. Curr Allergy Asthma Rep 18:77

Hong D, Kang Y, Brenner A et al (2015) MRX34, a liposomal miR-34 mimic, in patients with advanced solid tumours: final dose-escalation results from a first-in-human phase I trial of microRNA therapy. J Clin Oncol 34(suppl 15):2508

Hosgood HD, Menashe I, He X et al (2009) PTEN identified as important risk factor of chronic obstructive pulmonary disease. Respir Med 103:1866-1870

Hou B, Murata M, Said A et al (2015) Changes in micro-RNAs in asymptomatic subjects sensitized to Japanese cedar pollen after prophylactic sublingual immunotherapy. Allergy Rhinol 6:33-38

Hu R, Pan W, Fedulov A et al (2014) MicroRNA-10 controls airway smooth muscle cell proliferation via direct targeting of the PI3 kinase pathway. FASEB J 28:2347-2357

Hu D, Zhang Z, Ke X et al (2017) A functional variant of miRNA-149 confers risk for allergic rhinitis and comorbid asthma in Chinese children. Int J Immunogenet 44:62-70

Huang Y, Zhang S, Fang X et al (2018) Plasma miR-199a-5p is increased in neutrophilic phenotype asthma patients and negatively correlated with pulmonary function. PLoS One 13:e0193502

Huo X, Zhang K, Yi L et al (2016) Decreased epithelial and plasma miR-181b-5p expression associates with airway eosinophilic inflammation in asthma. Clin Exp Allergy 46:1281-1290

Janssen H, Reesink H, Lawitz E et al (2013) Treatment of HCV infection by targeting microRNA. N Engl J Med 368:1685-1694

Jardim M, Dailey L, Silbajoris R et al (2012) Distinct microRNA expression in human airway cells of asthmatic donors identifies a novel asthma-associated gene. Am J Respir Cell Mol Biol 47:536-542

Jen K, Campo M, He H et al (2007) CD45RB ligation inhibits allergic pulmonary inflammation by inducing CTLA4 transcription. J Immunol 179:4212-4218

Jia M, Chu C, Wang M (2018) Correlation of microRNA profiles with disease risk and severity of allergic rhinitis. Int J Clin Exp Pathol 11:1791-1802

Kariyawasam H, Xanthou G, Barkans J et al (2008) Basal expression of bone morphogenetic protein receptor is reduced in mild asthma. Am J Respir Crit Care Med 177:1074-1081

Karner J, Wawrzyniak M, Tankov S et al (2017) Increased microRNA-323-3p in IL-22/IL-17-producing T cells and asthma: a role in the regulation of the TGF- $\beta$ pathway and IL- 22 production. Allergy 72:55-65

Kim RY, Horvat JC, Pinkerton JW et al (2017) MicroRNA-21 drives severe, steroid-insensitive experimental asthma by amplifying phosphoinositide 3-kinase-mediated suppression of histone deacetylase 2. J Allergy Clin Immunol 139:519-532

Kuhn AR, Schlauch K, Lao R et al (2010) MicroRNA expression in human airway smooth muscle cells: role of miR-25 in regulation of airway smooth muscle phenotype. Am J Respir Cell Mol Biol 42:506-513

Kumar M, Ahmad T, Sharma A et al (2011) Let-7 microRNA-mediated regulation of IL-13 and allergic airway inflammation. J Allergy Clin Immunol 128(1077-1085):e1-10

Lacedonia D, Palladino GP, Foschino-Barbaro MP et al (2017) Expression profiling of miRNA-145 and miRNA-338 in serum and sputum of patients with COPD, asthma, asthma-COPD overlap syndrome phenotype. Int J Chron Obstruct Pulmon Dis 12:1811-1817

Lee R, Feinbaum R, Ambros V (1993) The C. elegans gene lin-4 encodes small RNAs with antisense complementarity to lin-14. Cell 75:843-854

Li Q, Chau J, Ebert P et al (2007) miR-181a is an intrinsic modulator of $\mathrm{T}$ cell sensitivity and selection. Cell 129:147-161

Li L, Zhang S, Jiang X et al (2018) MicroRNA-let-7e regulates the progression and development of allergic rhinitis by targeting suppressor of cytokine signalling 4 and activating Janus kinase 1- signal transducer and activator of transcription 3 pathway. Exp Ther Med 15:3523-3529

Liu F, Qin H, Xu B et al (2012) Profiling of microRNAs in pediatric asthma: upregulation of miRNA-221 and miRNA-485-3p. Mol Med Rep 6:1178-1182

Liu Y, Sun X, Wu Y et al (2015) Effects of miRNA-145 on airway smooth muscle cells function. Mol Cell Biochem 409:135-143

Liu H, Zhang A, Zhao N et al (2016a) Role of miR-146a in enforcing effect of specific immunotherapy on allergic rhinitis. Immunol Invest 45:1-10

Liu W, Zeng Q, Luo R (2016b) Correlation between serum osteopontin and miR-181a levels in allergic rhinitis children. Mediat Inflamm 2016:9471215

Long CM, Lukomska E, Marshall NB et al (2017) Potential inhibitory influence of miRNA-210 on regulatory T cells during epicutaneous chemical sensitization. Genes 8:E9 
Lu TX, Rothenberg ME (2013) Diagnostic, functional and therapeutic roles of microRNa in allergic diseases. J Allergy Clin Immunol 132:3-13

Lu TX, Munitz A, Rothenberg ME (2009) MicroRNA-21 is up-regulated in allergic airway inflammation and regulates IL-12p35 expression. J Immunol 182:4994-5002

Lu TX, Hartner J, Lim EJ et al (2011) MicroRNA-21 limits in vivo immune response-mediated activation of the IL-12/IFNgamma pathway, Th1 polarization and the severity of delayedtype hypersensitivity. J Immunol 187:3362-3373

Lu TX, Lim EJ, Besse JA et al (2013a) MiR-223 deficiency increases eosinophil progenitor proliferation. J Immunol 190:1576-1582

Lu TX, Lim EJ, Itskovich S et al (2013b) Targeted ablation of miR-21 decreases murine eosinophil progenitor cell growth. PLoS One 8:e59397

Luo Y, Deng Y, Tao Z et al (2014) Regulatory effect of microRNA$135 \mathrm{a}$ on the Th1/Th2 imbalance in a murine model of allergic rhinitis. Exp Ther Med 8:1105-1110

Luo X, Han M, Liu J et al (2015) Epithelial cell-derived microRNA146a generates interleukin-10 producing monocytes to inhibit nasal allergy. Sci Rep 5:15937

Luo X, Hong H, Tang J et al (2016) Increased expression od miR-146a in children with allergic rhinitis after allergen-specific immunotherapy. Allergy Asthma Immunol Res 8:132-140

Lv Y, Qi R, Xu J et al (2014) Profiling of serum and urinary microRNAs in children with atopic dermatitis. PLoS One 9:e115448

Maes T, Cobos FA, Schleich F et al (2016) Asthma inflammatory phenotypes show differential microRNA expression in sputum. $\mathrm{J}$ Allergy Clin Immunol 137:1433-1446

McClure C, Brudecki L, Fergusson DA et al (2014) MicroRNA 21 (miR-21) and miR-181b couple with NFI-A to generate myeloidderived suppressor cells and promote immunosuppression in late sepsis. Infect Immun 82:3816-3825

McGeachie MJ, Davis JS, Kho AT et al (2017) Asthma remission: predicting future airways responsiveness using an miRNA network. J Allergy Clin Immunol 140:598-600

Milger K, Gotschke J, Krause L et al (2017) Identification of a plasma miRNA biomarker signature for allergic asthma: a translational approach. Allergy 72:1962-1971

Niu R, Xiao X, Liu B et al (2017) Inhibition of airway inflammation in a cockroach allergen model of asthma by agonists of miRNA33b. Sci Rep 7:7409

Odhiambo JA, Williams HC, Clayton TO et al (2009) Global variations in prevalence of eczema symptoms in children from ISAAC Phase Three. J Allergy Clin Immunol 124:1251-1258 e.23

Oh SH, Park SM, Lee YH et al (2009) Association of peroxisome proliferator-activated receptor-gamma gene polymorphisms with the development of asthma. Respir Med 103:1020-1024

Panganiban RP, Pinkerton MH, Maru SY et al (2012) Differential microRNA expression in asthma and the role of miR-1248 in regulation of IL-5. Am J Clin Exp Immunol 1:154-165

Panganiban RP, Wang Y, Howrylak J et al (2016) Circulating microRNAs as biomarkers in patients with allergic rhinitis and asthma. J Allergy Clin Immunol 137:1423-1432

Parikh NI, Vasan RS (2007) Assessing the clinical utility of biomarkers in medicine. Biomark Med 1:419-436

Plank MW, Maltby S, Tay HL et al (2015) MicroRNA expression is altered in an ovalbumin-induced asthma model and targeting miR-155 with antagomirs reveals cellular specificity. PLoS One 10:e0144810

Polikepahad S, Knight JM, Naghavi AO et al (2010) Proinflammatory role for let-7 microRNAs in experimental asthma. J Biol Chem 285:30139-30149

Qin HB, Xu B, Mei JJ et al (2012) Inhibition of miR-221 suppresses the airway inflammation in asthma. Inflammation 35:1595-1599
Rebane A, Akdis CA (2014) MicroRNAs in allergy and asthma. Curr Allergy Asthma Rep 14:424

Reid G, Kao SC, Pavlakis N et al (2016) Clinical development of targomiRs, a miRNA mimic-based treatment for patients with recurrent thoracic cancer. Epigenomics 8:1079-1085

Sawant DV, Wu H, Kaplan MH et al (2013) The Bcl6 target gene microRNA-21 promotes Th2 differentiation by a $\mathrm{T}$ cell intrinsic pathway. Mol Immunol 54:435-442

Shaoqing Y, Ruxin Z, Guojun L et al (2011) Microarray analysis of differently expressed microRNAs in allergic rhinitis. Am J Rhinol Allergy 25:e-242-e-246

Simpson M, Brede G, Johansen J et al (2015) Human breast milk miRNA, maternal probiotic supplementation and atopic dermatitis in offspring. PLoS One 10:e143496

Skrzypski M, Czapiewski P, Goryca K et al (2014) Prognostic value of microRNA expression in operable non-small cell lung cancer patients. Br J Cancer 110:991-1000

Solberg OD, Ostrin EJ, Love MI et al (2012) Airway epithelial miRNA expression is altered in asthma. Am J Respir Crit Care Med 186:965-974

Sonkoly E, Janson P, Majuri ML et al (2010) Mir-155 is overexpressed in patients with atopic dermatitis and modulates T-cell proliferative responses by targeting cytotoxic $\mathrm{T}$ lymphocyte-associated antigen 4. J Allergy Clin Immunol 126(581-589):e1-20

Soujalehto H, Lindstrom I, Majuri ML et al (2014) Altered microRNA expression in long-term asthma and allergic rhinitis. Int Arch Allergy Immunol 163:168-178

Specjalski K, Maciejewska A, Pawłowski R et al (2016) Changes in the expression of microRNA in the buildup phase of wasp venom immunotherapy: a pilot study. Int Arch Allergy Immunol 170:97-100

Stenvang J, Petri A, Lindow M et al (2012) Inhibition of microRNA function by antimir oligonucleotides. Silence 3:1

Su XW, Yang Y, Lv ML et al (2011) Association between single-nucleotide polymorphisms in pre-miRNAs and the risk of asthma in Chinese population. DNA Cell Biol 30:919-923

Suojalehto H, Toskala E, Kipelainen M et al (2013) MicroRNA profiles in nasal mucosa of patients with allergic and nonallergic rhinitis and asthma. Int Forum Allergy Rhinol 3:612-620

Tang H, Jiang H, Zheng J et al (2015) MicroRNA-106b regulates proallergic properties of dendritic cells and $\mathrm{Th} 2$ polarization by targeting early growth response- 2 in vitro. Int Immunopharmacol 28:866-874

Teng Y, Zhang R, Liu C et al (2015) MiR-143 inhibits interleukin13 -induced inflammatory cytokine and mucus production in nasal epithelial cells from allergic rhinitis patients by targeting IL13R $\alpha 1$. Biochem Biophys Res Commun 457:58-64

Trinh HK, Pham DL, Kim SC et al (2017) Association of the miR196a, miR-146a and miR-499 polymorphisms with asthma phenotypes in a Korean population. Mol Diagn Ther 21:547-554

Wang WC, Juan AH, Panebra A et al (2011) MicroRNA let-7 establishes expression of beta2-adrenergic receptors and dynamically down-regulates agonist-promoted down-regulation. Proc Natl Acad Sci USA 108:6246-6251

Wang SB, Deng YQ, Ren J et al (2014) Exogenous interleukin-10 alleviates allergic inflammation but inhibits local interleukin-10 expression in a mouse allergic rhinitis model. BMC Immunol 15:9

Wang T, Chen D, Wang P et al (2018) MiR-375 prevents nasal mucosa cells from apoptosis and ameliorates allergic rhinitis via inhibiting JAK3/STAT3 pathway. Biomed Pharmacother 103:621-627

Weiss LA, Lester LA, Gern JE et al (2005) Variation in ITGB3 is associated with asthma and sensitization to mold allergen in four populations. Am J Respir Crit Care Med 172:67-73 
Williams AE, Larner-Svensson H, Perry MM et al (2009) MicroRNA expression profiling in mild asthmatic human airways and effect of corticosteroid therapy. PLoS One 4:e5889

Wollenberg A, Barbarot S, Bieber T et al (2018) Consensus-based European guidelines for treatment of atopic eczema (atopic dermatitis) in adults and children; part I. J Eur Acad Dermatol Venereol 32:657-682

Wu G, Yang G, Zhang R et al (2015) Altered microRNA expression profiles in extracellular vesicles in nasal mucus from patients allergic rhinitis. Allergy Asthma Immunol Res 7:449-457

Xiao R, Noel A, Perveen Z et al (2016) In utero exposure to secondhand smoke activates pro-asthmatic and oncogenic miRNAs in adult asthmatic mice. Environ Mol Mutagen 57:190-199

Xiao L, Jiang L, Hu Q et al (2017) MicroRNA-133b ameliorates allergic inflammation and symptom in murine model of allergic rhinitis by targeting NIrp3. Cell Physiol Biochem 42:901-912

Yang Y, Yin X, Yi J et al (2017) MiR-146a overexpression effectively improves experimental allergic conjunctivitis through regulating CD4 + CD25-T cells. Biomed Pharmacother 94:937-943

Yu S, Zhang R, Zhu C et al (2013) MircoRNA-143 downregulates interleukin-13 receptor alpha-1 in human mast cells. Int J Mol Sci 14:16958-16969
Zhang Y, Sun E, Li X et al (2017) MiR-155 contributes to Df1-induced asthma by increasing the proliferative response of Th cells via CTLA-4 downregulation. Cell Immunol 314:1-9

Zhao CY, Wang W, Yao HC et al (2018) SOCS3 in upregulated and targeted by MiR30a-5p in allergic rhinitis. Int Arch Allergy Immunol 175:209-219

Zhou H, Li J, Gao P et al (2016a) MiR-155: a novel target in allergic asthma. Int J Mol Sci 17:E1773

Zhou Y, Yang Q, Xu H et al (2016b) miRNA-221-3p enhances the secretion of interleukin-4 in mast cells through the phosphatase and tensin homolog (p38) nuclear factor-kappa $\beta$ pathway. PLoS One 11:e0148821

Publisher's Note Springer Nature remains neutral with regard to jurisdictional claims in published maps and institutional affiliations. 